\title{
Diseño y documentación del Sistema de Gestión de la Seguridad y Salud en el Trabajo en la empresa Agregados de Sucre S. A. S., ubicada en el municipio de Toluviejo, Sucre
}

\section{Resumen}

Sandra Molina', Fabián Cruz²

A través del presente trabajo, se realizó el diseño y documentación del Sistema de Gestión de la Seguridad y Salud en el Trabajo en la empresa Agregados de Sucre S.A.S, ubicada en el municipio de Toluviejo, Sucre, según lo establecido en el Decreto 1072 del 2015 en su libro 2 parte 2 título 4 capítulo 6 y los estándares mínimos del Sistema de Gestión de la Seguridad y Salud en el Trabajo establecidos en la Resolución 1111 del 27 de marzo del 2017 para empleadores y contratantes. Como resultado se documentaron las herramientas necesarias para la implementación de este sistema de gestión por parte de la gerencia de la empresa para así disminuir los riesgos laborales dentro de la operación minera y todas las actividades relacionadas con la extracción de la caliza. Se identificaron, evaluaron y valoraron los peligros y riesgos basados en las normas GTC 45, con el fin de tomar las acciones correctivas y preventivas y de evitar futuros accidentes, teniendo en cuenta los indicadores del sistema en los que se refleja la ocurrencia de accidentes, incidentes, enfermedad y ausentismos de tipo laboral.

Palabras clave: incidentes y accidentes, riesgos laborales, Sistema de Gestión de la Seguridad y Salud en el Trabajo.

\footnotetext{
1 Ingeniera de Minas de la Fundación Universitaria del Área Andina. Valledupar, Colombia. Correo: Smolina17@areandina.edu.co

2 Estudiante de la Fundación Universitaria del Área Andina. Valledupar, Colombia. Facultad de Ingeniería y Ciencias Básicas. Correo: fcruz5@estudiantes.areandina.edu.co
} 


\section{Introducción}

El Sistema de Gestión de la Seguridad y Salud en el Trabajo (SG-SST) se ha vuelto prioridad con el pasar de los años debido a la importancia que genera la seguridad y salud de los empleados en las empresas, además de cumplir con las obligaciones que tiene la empresa con sus trabajadores y velar por un espacio seguro para ejercer su labor y los impactos negativos que genera el no contar con un sistema de gestión y la no aplicación de dicho sistema en mejora de la seguridad de su operación.

En Agregados de Sucre S.A.S no existe dicho sistema, lo que trae consigo el manejo inadecuado de eventualidades en materia de seguridad laboral, como la falta de conocimientos al momento de que estas eventualidades se presentan.

Esta situación ocasiona que se presenten accidentes e incidentes en los entornos laborales y que no se manejen de manera adecuada para identificar las causas de estos hechos; adicionalmente, no existe evidencia física de los procedimientos adecuados para una operación segura, tampoco una matriz de riesgo o un plan de emergencia. No se tiene conformado un COPASST ni un comité de convivencia laboral, no se realizan capacitaciones, entre otras carencias.

Para enfrentar estas situaciones se estructurará un plan de acción que permita realizar las actividades pendientes, basándose en el diagnóstico inicial de la empresa al momento de comenzar el proyecto con respecto a los requisitos mínimos exigidos por la Resolución 1111 de 2017, incluyendo entre estos una matriz de riesgo que permita identificar, evaluar y valorar los peligros y riesgos, además de poder verificar la implementación de las acciones correctivas, creando así los procedimientos de seguridad adecuados para cada una de las operaciones dentro de la empresa y establecer indicadores que reflejen la ocurrencia de accidentes, incidentes, enfermedades y ausentismos de tipo laboral, con la finalidad de mejorar estos indicadores. 


\section{Materiales y métodos}

\section{Localización}

La empresa Agregados de Sucre S. A. S. se encuentra ubicada a $5 \mathrm{~km}$ del municipio de Toluviejo, vía Toluviejo - San Onofre, Sucre (ver figura 1).

Figura 1. Ubicación geografía de la empresa Agregados de Sucre S. A.S

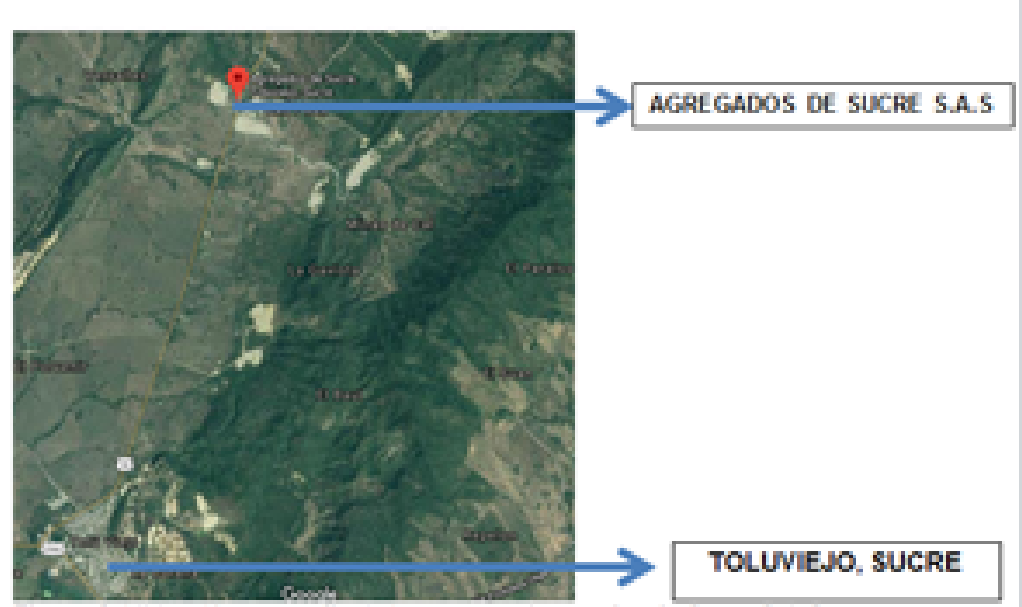

Fuente: tomada de Google Maps.

\section{Tipo de investigación}

El tipo de investigación que se llevara a cabo en este proyecto es de carácter cualitativo, en la cual se realizará el diseño teniendo en cuenta lo establecido en el Decreto 1072 en su libro 2 parte 2 titulo 4 capítulo 6 , comenzando por una evaluación

inicial o el diagnóstico inicial de la empresa, tomando como metodología lo definido por la Resolución 1111 de 2017, la cual define los estándares mínimos del
Sistema de Gestión de la Seguridad y Salud en el Trabajo que tiene como objeto "implementar los estándares mínimos del sistema de gestión de seguridad y salud en el trabajo para las entidades, personas o empresas señaladas en el campo de aplicación de la presente resolución".

\section{Procedimiento}

Para la identificación, evaluación y valoración de los peligros y riesgos se tendrá en cuenta como metodología la 
definida por la GTC 45, la cual es una "guía que proporciona directrices para identificar los peligros y valorar los riesgos en salud y seguridad en el trabajo, teniendo en cuenta la naturaleza de la empresa, el alcance de sus actividades y los recursos establecidos"

(Icontec, 2012); para controlar estos riesgos se realizaron procedimientos de seguridad, reportes de actos y condiciones inseguras y se establecieron indicadores que reflejan la ocurrencia de accidentes basados en los indicadores mínimos de seguridad y salud en el trabajo en la Resolución 1111 de 2017 en el artículo 15 , los cuales se indican en la tabla 1 .

Tabla 1. Indicadores mínimos de seguridad y salud en el trabajo

\begin{tabular}{|l|l|l|l|}
\hline Nombre del indicador & Definición & Fórmula & Periocidad Mínima \\
\hline $\begin{array}{l}\text { Severidad de los accidentes } \\
\text { laborles }\end{array}$ & & & Anual \\
\hline $\begin{array}{l}\text { Frecuencia de los accidentes } \\
\text { laborles }\end{array}$ & & & Anual \\
\hline $\begin{array}{l}\text { Mortalidad de los accidentes } \\
\text { laborles }\end{array}$ & & & Anual \\
\hline $\begin{array}{l}\text { Prevalencia de los acciden- } \\
\text { tes laborles }\end{array}$ & & & Anual \\
\hline $\begin{array}{l}\text { Incidencia de los accidentes } \\
\text { laborles }\end{array}$ & & & Anual \\
\hline Ausentismo & & & Anual \\
\hline
\end{tabular}

Fuente: Resolución 1111 de 2017. Artículo 15

Además de preservar la integridad del empleado con dicho sistema, se busca así cumplir con los requisitos establecidos en el libro 2 parte 2 título 4 capítulo 6 del Decreto 1072 de 2015 y Resolución 1111 del 2017 por el Ministerio del Trabajo, en los que se establecen los estándares mínimos que deben cumplir las empresas de forma obligatoria.

\section{Resultados}

Este proyecto de grado es de carácter cualitativo, el cual documentó el Sistema de Gestión de la Seguridad y Salud en el Trabajo, cumpliendo con los estándares mínimos de la Resolución 1111 de 2017, el Decreto 1072 de 2015 y con los objetivos del proyecto, con la evaluación inicial del estado en el que se encontraba la empresa con respecto a 
lo exigido por la norma, siguiendo con la identificación de riesgos con la matriz de peligros, valoración de riesgos y determinación de controles para así contar con los procedimientos adecuados de las operaciones dentro de la empresa y por medio de los indicadores de estructura, proceso y de resultado poder medir y realizar un seguimiento de los resultados de la implementación del sistema de gestión.

- Evaluación inicial

Con la realización de la evaluación inicial se evidencio que la empresa se encuentra en estado crítico, pues tiene un $7 \%$ de cumplimiento con lo exigido por la normatividad colombiana; al finalizar la dejamos con un $41,7 \%$, pues no se realizó la implementación del sistema.

- Matriz de peligros, valoración de riesgos y determinación de controles

Se realizó la matriz de peligros teniendo en cuenta el proceso operativo de la empresa con base en el lugar (planta de producción o cantera) donde se realiza la actividad (extracción de materia prima, beneficio de agregados, transporte de materia prima y mantenimiento preventivo o correctivo).

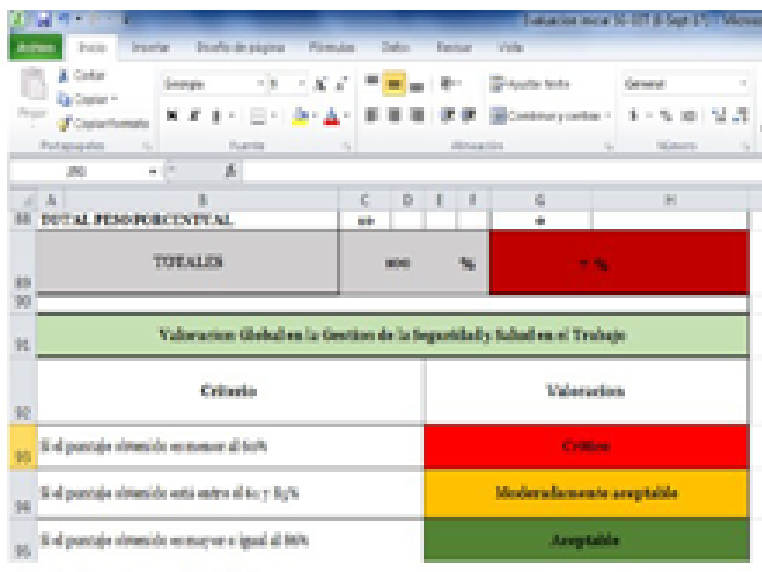

forte: fonwepe istr (ath)

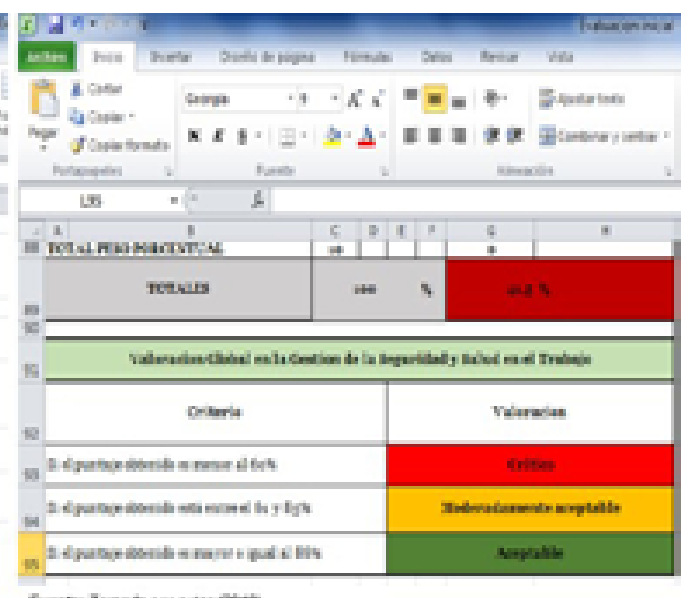

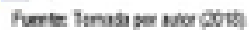

Evaluación inicial de la empresa.

Figura 2. 
- Procedimientos internos de seguridad

Se realizaron procedimientos para cumplir con la futura implementación del sistema por parte de la empresa, como también de seguridad para disminuir el riesgo presente en las tareas principales de la operación minera. Dentro de los procedimientos que se documentaron se encuentran el procedimiento de cargue en cantera y procedimiento para iniciar la planta trituradora.

- Indicadores del SG-SST

Se tuvieron en cuenta unos indicadores para medir la ocurrencia de accidentes y controlar las eventualidades que se presenten durante la operación. Dentro de los controles de seguimiento se establecieron los siguientes indicadores:

- Número de trabajadores directos.

- Número de trabajos de contratistas

- Número de accidentes reportados en el periodo.

\section{Conclusiones}

Con este proyecto se puede concluir que se cumplió con los estándares mínimos de seguridad para la empresa Agregados de Sucre S.A.S., la cual cuenta con la documentación necesaria para vigilar las operaciones y controlar los riesgos presentes en cada una de las actividades.

Es necesario que exista un compromiso por parte de la gerencia para que este sistema funcione, es por esto que se debe ir implementando paulatinamente el sistema de gestión y así hacer uso de la documentación en busca de mejorar la seguridad, la salud y la calidad de vida del trabajador, como también reducir costos y periodos de ausentismo que son generados por accidentes, lo que tiene como consecuencia el retraso en las operaciones. Es por esto que la empresa ha tomado como inicio de sus mediciones los 6 indicadores que exige la Resolución 1111 de 2017 (ver tabla 1), de igual forma ha realizado las inspecciones documentadas dentro del sistema (ver anexo inspecciones) y la conformación de COPASST y del comité de convivencia laboral y sus respectivas reuniones.

Con este proyecto se evidenció la importancia de la seguridad y la salud de los trabajadores para la empresa Agregados de Sucre S.A. S., la cual fue mostrando interés al avanzar este proyecto con el fin de ser una de las empresas mejores posicionadas en la región en todos los ámbitos, no solo minero y comercial, sino también mostrar su responsabilidad con sus empleados para así ser ejemplo 
de las empresas mineras de la región.

\section{Recomendaciones}

Teniendo en cuenta el desarrollo del presente proyecto basado en el Decreto 1072 del 2015 y la Resolución 1111 de 2017, se recomienda que para implementar el SG-SST la empresa debe contar con un profesional idóneo para el manejo adecuado del sistema (artículo 6 numeral 6.2 parágrafo 1 y 2 de la Resolución 1111) procedimental en todo tipo de actividad nueva que represente un riesgo en la salud y en la condición de trabajo de los empleados, además de realizar la socialización de estos; también socializar cualquier cambio que se genere en los que ya se dejaron documentados dentro del sistema de gestión (SG-SST). También es importante que los empleadores fomenten el buen uso de los elementos de protección personal como el realizar capacitaciones orienta- das a concientizar al trabajador del riesgo al cual está expuesto en su trabajo cotidiano, buscando así reducir el número de accidentes y estimulando el autocuidado por parte de cada empleado dentro de sus operaciones en la empresa.

\section{Referencias}

Ministerio del Trabajo (2017). Decreto 1072 de 2015 libro 2, parte 2, título 4, capítulo 6. Recuperado de http://www.mintrabajo.gov. co/documents/20147/50711/DUR+Sector + Trabajo + Actualizado $+\mathrm{a}+$ Octubre + $\mathrm{de}+2017$.pdf/e85f8008-7886-2d70-f894$112075 \mathrm{f} 0 \mathrm{c} 8 \mathrm{da}$

Ministerio del Trabajo (2017). Resolución 1111 de 2017. Recuperado de http://www.mintrabajo.gov.co/documents/20147/647970/ Resoluci\%C3\%B3n+1111-+est\%C3\%A1ndares + minimos-marzo +27 .pdf

Instituto Colombiano de Normas Técnicas y Certificación (Icontec). GTC 45 de 2012. Recuperado de http://idrd.gov.co/sitio/idrd/ sites/default/files/imagenes/gtc450.pdf 